\title{
Simulation Study of Frequency Variations of VLF Triggered Emissions in a Homogeneous Field
}

\author{
Yoshiharu OMURA ${ }^{1}$ and Hiroshi MATSUmoto ${ }^{2}$ \\ ${ }^{1}$ Department of Electrical Engineering, Kyoto University, Kyoto, Japan \\ ${ }^{2}$ Radio Atmospheric Science Center, Kyoto University, Kyoto, Japan \\ (Received November 30, 1984; Revised May 22, 1985)
}

\begin{abstract}
The whistler mode wave-particle interaction in a homogeneous magnetic field is studied by computer simulation. A monochromatic whistler wave packet propagating parallel to the magnetic field is assumed to interact with counter streaming high energy electrons. Main emphasis is laid on the following points: (1) whether or not the emission is triggerable within the homogeneous model, (2) if so, how much the frequency deviate from the triggering frequency and (3) what is the physical mechanism of the frequency deviation. It is demonstrated that the new emission is triggered owing to detrapping of phase-bunched electrons from the pulse. The emission shows a relatively small rising tone followed by subsequent frequency oscillations. The range of the frequency variation is about $5 \%$ of the triggering pulse. This is supposed to correspond to the "embryo" emission phase or to the initial phase of triggering of emissions.
\end{abstract}

\section{Introduction}

Whistler pulses of $50 \sim 250 \mathrm{msec}$ transmitted from the Siple station in Antarctica into the magnetosphere are found to be accompanied by narrowband VLF triggered emissions with a variety of nonlinear frequency variation (HELliWELL and KATSUFRAKIS, 1974). It is generally believed that these VLF emissions originate from the region close to the equatorial plane of the magnetosphere owing to a cyclotron resonance with counter-streaming high energy electrons. Many numerical simulation studies as well as theoretical ones are reported so far. (See a review by Matsumoto (1979)). None of the computer simulations, however, have ever succeeded in reproducing nonlinear frequency changes of the triggered emissions as observed in the magnetosphere.

In this paper we present a computer simulation of coherent whistler waveparticle interaction in which a whistler wave pulse propagating along an external magnetic field interacts with counter-streaming high energy electrons in the homogeneous magnetic field which may well model the equatorial region. Several simulations similar to this model were performed by several authors. (DENAVIT and SudAn, 1975; VomVORIDIS, 1978; OMURA and MATSUMOTO, 1982; HaSHIMOTO et al., 1983). Among these simulation studies, only DENAvit and SudAN (1975) 
report formation of new emissions with rapid frequency variations in their homogeneous model. However, the parameters they assumed are unrealistic and quite different from those in the magnetospheric conditions. VOMVORIDIS (1978) also studied the emissions in a nonuniform magnetic field. He found that only a small frequency shift could happen even in his nonuniform model. However, it is not clear whether the frequency shift is due to the nonuniformity of the magnetic field or due to to the interaction intrinsic to the homogeneous model. Assuming the magnetospheric parameters, we also found a generation of a new wave packet, i.e., a triggered emission in our homogeneous model. The emission shows a relatively small frequency variations, i.e., about $5 \%$ of the triggering wave frequency. We interpret the emission observed in the present simulation is the indication of the initial phase "embryo" emission (DOWDEN et al., 1978) of VLF triggered emissions. We give a new physical interpretation that a rising tone is caused by detrapping of phase bunched electrons and that small frequency shifts are possible even in a uniform magnetic field. Following the introduction, the simulation model is described in Section 2. The results of the simulations and their interpretation are presented in Section 3. Section 4 gives a summary and discussion.

\section{Simulation Model}

We assume a purely transverse whistler mode wave that propagates along the geomagnetic field line and that interacts with counter-streaming high energy electrons from its wave front first. The evolution of the wave amplitude and frequency are governed by the following equations (OMURA and MATSUMOTO, 1982).

$$
\begin{aligned}
\frac{\partial B_{\mathrm{w}}}{\partial t} & =-V_{\mathrm{g}}\left(\frac{\partial B_{\mathrm{w}}}{\partial z}+\frac{1}{2} \mu_{0} J_{E}\right) \\
\frac{\partial k}{\partial t} & =-\frac{\partial \omega}{\partial z} \\
\omega & =\frac{k\left(k-\mu_{0} J_{B} / B_{\mathrm{w}}\right)}{k\left(k-\mu_{0} J_{B} / B_{\mathrm{w}}\right)+\Pi_{e}^{2} / c^{2}} \Omega_{\mathrm{e}}
\end{aligned}
$$

where $V_{\mathrm{g}}$ are the group velocity given by

$$
V_{\mathrm{g}}=2 k\left(\Omega_{\mathrm{e}}-\omega\right) /\left(k^{2}+\Pi_{\mathrm{e}}^{2} / c^{2}\right)
$$

and $B_{\mathrm{w}}, \omega, k, \Omega_{\mathrm{e}}$ and $c$ are the wave amplitude, frequency, wavenumber, electron cyclotron frequency and light speed, respectively. $J_{E}$ and $J_{B}$ are the components of the transverse resonant currents parallel to the wave electric field $E_{\mathrm{w}}$ and to the wave magnetic field $B_{\mathrm{w}}$, respectively. Equations (1), (2) and (3) are solved by a difference scheme using the quantities defined at grid points. $J_{E}$ and $J_{B}$ are calculated by the following equations 


$$
J_{E}=-e \sum_{i} v_{\perp \mathrm{i}} \sin \left(-\zeta_{\mathrm{i}}\right), \quad J_{B}=-e \sum_{i} v_{\perp \mathrm{i}} \cos \left(-\zeta_{\mathrm{i}}\right)
$$

where $v_{\perp \mathrm{i}}$ is a perpendicular velocity component to the external magnetic field and $\zeta_{\mathrm{i}}$ is a phase angle between $v_{\perp}$ and $B_{\mathrm{w}}$, and $\Sigma$ is taken over resonant electrons in a region between two adjacent grid points.

The simulation parameters are chosen as follows, reflecting a magnetospheric condition at the equatorial region of $L=4$.

- Electron cyclotron frequency: $14 \mathrm{kHz}$.

- Electron plasma frequency: $140 \mathrm{kHz}$.

- Amplitude of the whistler pulse: 5 pT.

- Frequency of the whistler pulse: $7 \mathrm{kHz}$.

- Length of the whistler pulse: $700 \mathrm{~km}$.

- Group velocity of the whistler pulse: $15000 \mathrm{~km} / \mathrm{sec}$.

- Drift velocity of energetic electrons: $-15000 \mathrm{~km} / \mathrm{sec}$.

- Parallel temperature of energetic electrons: $57 \mathrm{eV}$.

- Perpendicular temperature of energetic electrons: $7.4 \mathrm{keV}$.

- Density ratio of energetic and cold electrons: $8 \times 10^{-7}$.

The cold electrons are not treated as particles but as a fluid which gives the plasma dispersion assumed in Eqs. (1) and (3). A finite length of a whistler wave packet is initially placed in the simulation region as seen in Fig. 1 where the spatial profile of the wave packet and its time evolution are plotted. The wave front with Gaussian shape is initially placed at $Z=1000 \mathrm{~km}$. It propagates toward the positive $z$ direction. The amplitude of the wave $B_{\mathrm{w}}$ is $10^{-5} B_{0}$ where $B_{0}$ is the external magnetic field. The simulation region consists of 101 grid points with a spacing of $20 \mathrm{~km}$. The energetic electrons are initially placed in the region $z \geqslant 1000 \mathrm{~km}$ and move toward the negative $z$ direction. Newly coming particles are subsequently injected at the left side boundary of the simulation region. In each cell 400 particles are placed on the average to represent a velocity distribution of energetic electrons which is a shifted Maxwellian distribution in $v_{/ \prime}$ and a subtracted Maxwellian realizing a loss cone distribution in $v_{\perp}$ (AsHouRAbDalla and KenNel, 1978), of a form of

$$
\begin{aligned}
& f\left(v_{/}, v_{\perp}\right)=A \exp \left\{-\left(\frac{v_{/ /}-V_{/ / 0}}{V_{/ / \mathrm{T}}}\right)^{2}\right\} g\left(v_{\perp}\right) \\
& g\left(v_{\perp}\right)=\frac{1}{1-\beta}\left\{\exp \left(-\frac{v_{\perp}^{2}}{V_{\perp \mathrm{T}}^{2}}\right)-\exp \left(-\frac{v_{\perp}^{2}}{\beta V_{\perp \mathrm{T}}^{2}}\right)\right\}
\end{aligned}
$$

where $V_{/ / 0}$ and $V_{\mathrm{T}}$ are chosen as $V_{/ / 0}=V_{\mathrm{R}}=0.05 c$ and $V_{\mathrm{R}}$ is the resonance velocity $\left(\omega-\Omega_{\mathrm{e}}\right) / k . \quad V_{/ / \mathrm{T}}=0.5 V_{\mathrm{t}}=2.2 \times 10^{-4} c$, where $V_{\mathrm{t}}$ is a trapping velocity $2 \sqrt{\left(v_{\perp 0} \Omega_{\mathrm{w}}\right) / k}$ in which $\Omega_{\mathrm{w}}=e B_{\mathrm{w}} / m$, and $e$ and $m$ are electronic charge and mass, respectively. $V_{\perp \mathrm{T}}$ and $\beta$ are chosen as $V_{\perp \mathrm{T}}=0.029 \mathrm{c}$ and $\beta=0.5$ so that the average perpendicular velocity $v_{\perp 0}$ is $0.05 \mathrm{c}$, and that the average pitch angle of the electrons is $45^{\circ}$. 


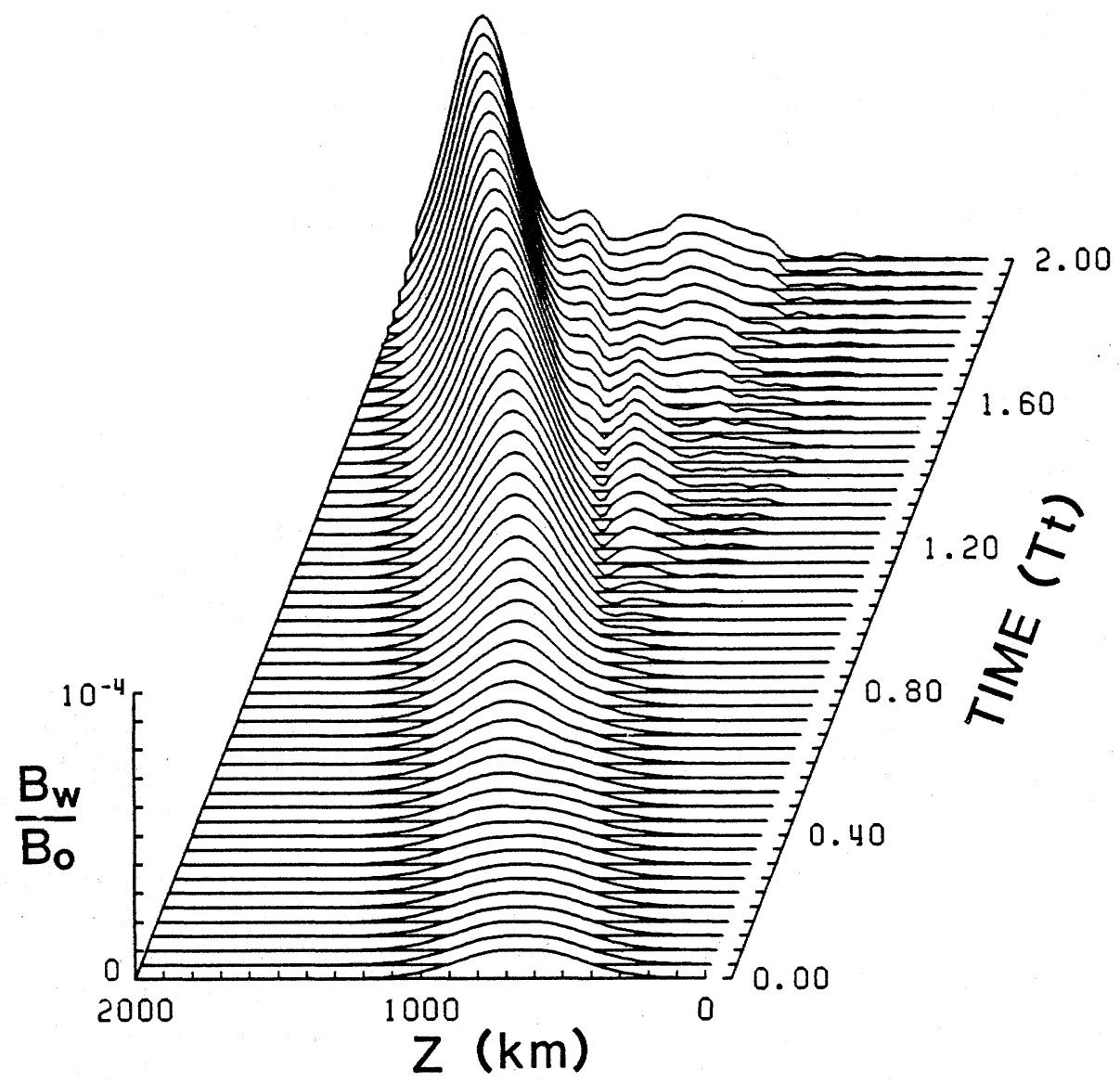

Fig. 1. Spatial profile of amplitude of a whistler packet and its time evolution. The whistler wave packet propagating to the positive $z$ direction is amplified and followed by a triggered emission generated at the rear end of the main pulse.

\section{Simulation Results and Their Interpretation}

The spatial profiles of the wave amplitude and its time evolution are shown in Fig. 1. The time is normalized by the trapping period $T_{\mathrm{t}}=2 \pi / \sqrt{k v_{\perp 0}\left(B_{\mathrm{w}} e / m\right)}$ calculated from the initial parameters. Under the present parameters, $T_{\mathrm{t}}$ corresponds to 447 electron cyclotron periods $(\sim 32 \mathrm{msec})$. The front portion of the pulse continuously grows receiving free energy from newly coming fresh electrons through cyclotron resonance. We find that the emission is emanating from the rear end of the triggering pulse. This triggered pulse is emitted from the resonant current formed by resonant electrons which have been trapped and phase bunched by the triggering pulse. 
The dynamic frequency spectra observed at $z=1000 \mathrm{~km}$ and at $z=1200 \mathrm{~km}$ are shown in Fig. 2. The amplitude of the wave is indicated by the width of the frequency spectra. The frequency of the triggering pulse shows a slight decrease first. When the amplitude decreases at the end of the triggering pulse, the frequency shows a small falling tone and turns to a rising tone followed by frequency oscillations. The portion which shows rising tone and subsequent oscillations corresponds to a triggered emission.

As expressed in Eq. (3), the frequency is controlled by the resonant current $J_{B}$ and $B_{\mathrm{w}}$ through a factor of $J_{B} / B_{\mathrm{w}}$, while the amplitude $B_{\mathrm{w}}$ is modified by $J_{E}$ as expressed in Eq. (1). The resonant currents are formed through the process of trapping, phase bunching and detrapping of resonant electrons. In order to analyze the process in detail, we plotted the local amplitude of the wave and the phase diagram of resonant electrons in the $v_{/ /} \zeta$ phase space at different times. Figure 3 shows the phase diagram of resonant electrons in the range of $z=1050 \sim 1150 \mathrm{~km}$. The effect of the wave is indicated by the separatrix of the trapping region whose range of $V_{/ /}$is given as

$$
V_{\mathrm{R}}-V_{\mathrm{t}} \leqslant v_{\| /} \leqslant V_{\mathrm{R}}+V_{\mathrm{t}}
$$

where $V_{\mathrm{t}}=2 \sqrt{\left(v_{\perp 0} \Omega_{\mathrm{w}}\right) / k}$. The vertical movement of the separatrix indicates the
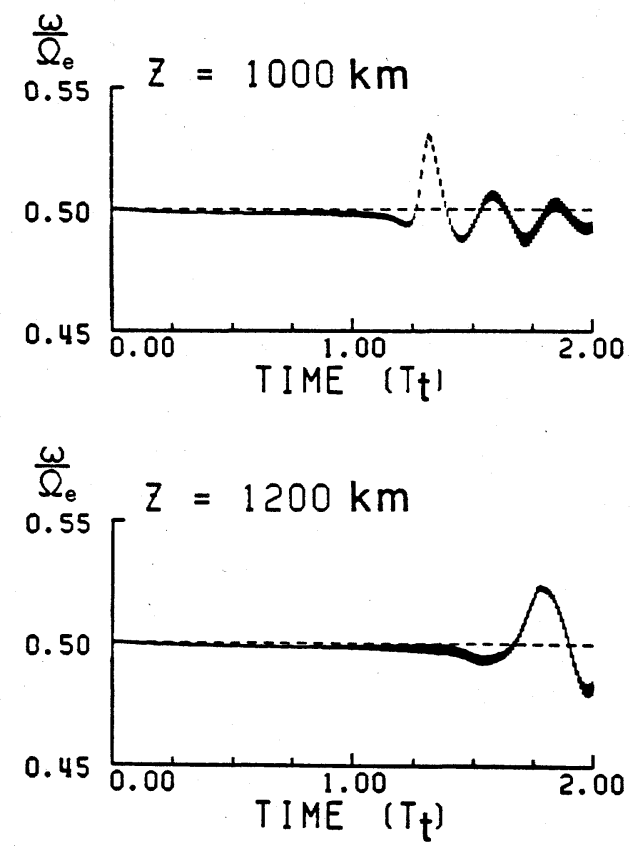

Fig. 2. Dynamic frequency spectra observed at $z=1000$ and $1200 \mathrm{~km}$. The width of the frequency spectra indicates the wave amplitude. 

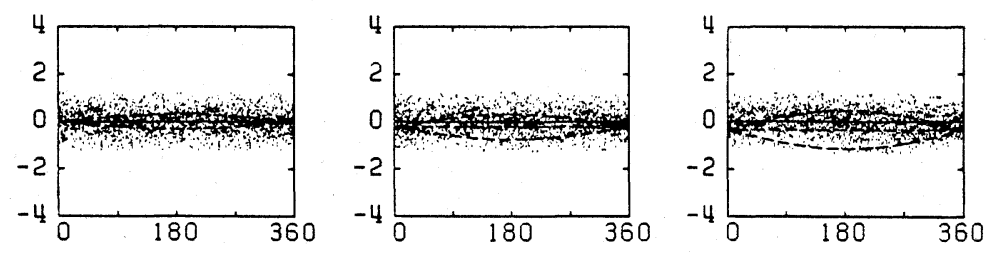

0.00

0.20

0.40
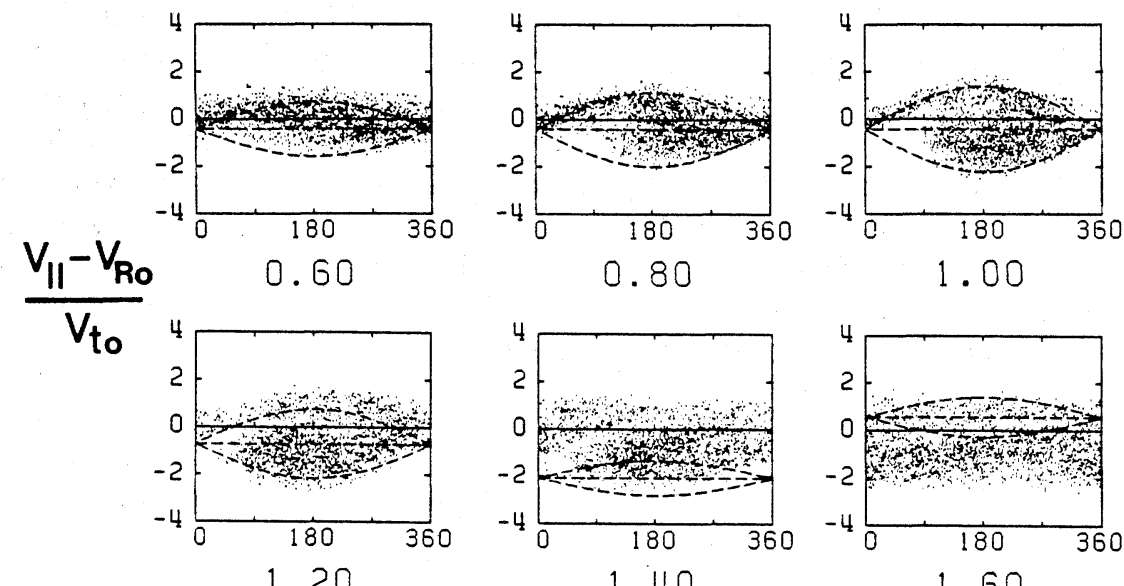

1.00

1.20

1.40

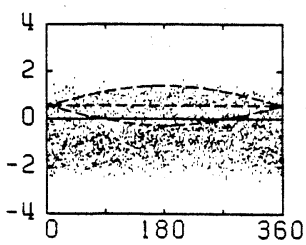

1.60

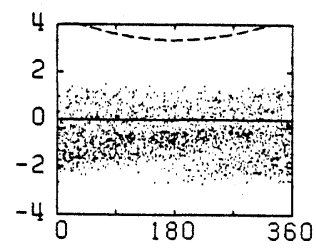

1.80

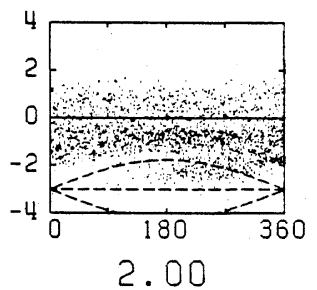

\section{$Z=1050$}

\section{$\sim 1150 \mathrm{~km}$}

Fig. 3. Phase diagrams of resonant electrons in the $v_{/ /} \zeta$ space at $z=1050 \sim 1150 \mathrm{~km}$. Numbers at the vertical axis indicate difference of $v_{\prime \prime}$ from the initial resonance velocity $V_{\mathrm{R}_{0}}$ normalized by the initial trapping velocity $V_{\mathrm{t}_{0}}$. Numbers below the phase diagrams indicate the trapping time. Eye-shaped dashed lines indicate the separatrices of trapping regions.

change of resonance velocity and correspond to the change of the frequency and wavenumber. The change of the size of the trapping region corresponds to the amplitude variation.

Before encountering the wave packet, electrons are distributed uniformly in phase. At $t=0.4 \sim 0.6$ the electrons are being engulfed by the front portion of the wave packet. Owing to the cyclotron resonance with the wave, the electrons trapped in the wave potential well begin a spiral motion around the center 
of the trapping region $\left(v_{1 /}, \zeta\right)=\left(V_{\mathrm{R}}, 180^{\circ}\right)$. Since the trapped electrons populate around the phase angle $180^{\circ}$, they form the positive resonant current $J_{B}$ and cause the frequency decrease, which is recognized from the downward motion of the separatrix in the phase diagram. Although the electrons are initially distributed symmetrically around the center of the trapping region, the symmetry breaks owing to the frequency decrease. This results in the phase bunching in the phase range of $\zeta=180^{\circ} \sim 360^{\circ}$ at $T=0.8 \sim 1.0$, which forms a negative $J_{E}$ causing the wave growth. The wave growth is recognized from the enlargement of the separatrix. The phase bunched electrons still continue to rotate in the potential well and fall in the phase range of $\zeta=0^{\circ} \sim 180^{\circ}$ at $T=1.2$, forming the positive $J_{E}$. Since the positive $J_{E}$ causes the wave damping, the trapping region shrinks and the phase bunched electrons are then detrapped from the potential well.

It is noted that the wave damping leads to the frequency decrease in the presence of the positive $J_{B}$. Therefore, the detrapping is induced by the frequency decrease as well as the wave damping. At $T=1.4$ we can observe the detrapping due to the both effects. Once detrapped from the potential well, the phase bunched electrons change their phase $\zeta$ with the rate as

$$
\frac{\mathrm{d} \zeta}{\mathrm{d} t}=k\left(v_{1 /}-V_{\mathrm{R}}\right)
$$

When the electrons comes to the phase range of $\zeta=-270^{\circ}$ to $90^{\circ}$, they form the positive $J_{B}$ causing the frequency increase, which corresponds to the rising tone in Fig. 2. As far as the phase correlation is retained, the detrapped electrons form resonant currents $J_{E}$ and $J_{B}$ whose signs change rapidly in time. These resonant currents cause the subsequent oscillations of the frequency and amplitude after the rising tone.

The interpretation stated above is not complete, for we have to consider the propagation effects as well. The changes of the amplitude and frequency observed at $z=1200 \mathrm{~km}$ are not the simple reflection of the local interaction as stated above, but they include those induced in the upstream region, as is obvious from the comparison between the spectra observed at $z=1000 \mathrm{~km}$ and $z=1200 \mathrm{~km}$. Also, the rapid frequency decrease indicated by the movement of the trapping region during the period of $1.8 \sim 2.0$ in Figure 3 cannot be explained only by the local resonant current induced by the detrapped electrons slightly phase-bunched. Basically, however, the resonant electrons undergo the above process of trapping, phase bunching and detrapping, causing the frequency decrease, wave growth, wave damping, rising tone and subsequent frequency oscillations.

\section{Summary and Discussion}

The computer simulation described in the preceding section showed an evolution of a monochromatic whistler wave pulse encountering counter streaming hot electrons in a homogeneous magnetic field. Our findings in the simulation 
are the following.

1) The front portion of the triggering pulse grows continuously receiving energy from resonant electrons which are freshly engulfed at the wave front.

2) Following the triggering pulse, an emission is generated by hot electrons which have been phase bunched by the triggering pulse.

3) The frequency of the triggering pulse decreases slightly due to the formation of a positive resonant current $J_{B}$. The decrease further leads to a small falling tone with a diminishing amplitude at the end of the triggering pulse. The latter portion is the initiation of the triggered emission.

4) The diminishing wave amplitude with the falling tone causes a detrapping of phase bunched electrons, resulting a rising tone and subsequent frequency oscillations. This process may correspond to the embryo emission discussed by DOWDEN et al. (1978).

5) The range of the frequency variation is about $5 \%$ of the initial wave frequency within the model of homogeneous magnetic field.

6) The time scale of the frequency variation is a fraction of the trapping period corresponding to $\sim 10 \mathrm{msec}$.

The range of frequency variations found in the present simulation is smaller, and the time scale of the variations is shorter than that of a typical triggered emission observed in the magnetosphere, which often exceeds $100 \%$ frequency variation. This is natural from the fact that detrapped electrons remain at a constant parallel velocity in the homogeneous magnetic field, and that the resonance condition $\omega=k_{v / /}+\Omega_{\mathrm{e}}$ remains constant. However, the present simulation revealed that the seed of the triggered emission and the initial phase of emission release is possible even in the homogeneous magnetic field, i.e., in the vicinity of the equatorial region. Also a physical mechanism of the emission release is clarified by the phase-plot examination. The frequency can be changed only by a resonant current $J_{B}$. Especially, a rising tone $J_{B}$ must be negative, which is impossible if the resonant electrons are stably trapped in a wave potential well. Therefore, detrapping of phase-bunched electrons are necessary for a rising tone (Roux and Pellat, 1978; MAtsumoto and OMURA, 1983). In the inhomogeneous geomagnetic field the rising tone could continue for a longer period, because the phase-bunched electrons, if detrapped at the equator, increase their parallel velocities $v$ /'s $(\leqslant 0)$. The inhomogeneity of the magnetic field seems to be indispensable for a wider and longer frequency variation. In order to include the inhomogeneity effect it is also indispensable to widen the velocity distribution function. This widening of $f\left(v_{/ /}\right)$is important even in the model of the homogeneous magnetic field, because the current simulation model postulated a beam type of resonant electrons.

Another point which needs to be commented is that only a single monochromatic whistler wave is treated in the present system. The frequency oscillation after the rising tone may reflect a situation where side bands are excited. A full electromagnetic particle simulation code can only treat the situation properly. The electromagnetic simulation with an inhomogeneous magnetic field 
is currently being undertaken in our group, and will be published elsewhere.

We would like to thank I. Kimura for his valuable discussion. This work was supported by Grant-in-Aid for Scientific Research of Monbusho of Japan No. 56420016. Suggestions by referees are appreciated.

\section{REFERENCES}

Ashour-Abdalla, M. and C. F. Kennel, Nonconvective and convective cyclotron harmonic instabilities, J. Gęophys. Res., 83, 1531-1543, 1978.

DENAVIT, J. and R. N. SUDAN, Effect of phase-correlated electrons on whistler wave packet propagation, Phys. Fluids, 18, 1533-1541, 1975.

Dowden, R. L., A. D. MCKAy, L. E. S. Amon, H. C. Koons, and M. H. DAzey, Linear and Nonlinear Amplification in the Magnetosphere During a 6.6-kHz Transmission, J. Geophys. Res., 83, 169-181, 1978.

Hashimoto, K., H. Matsumoto, Y. Serizawa, and I. KimuRa, Computer simulation of whistler mode wave-particle interactions using a free-boundary model, J. Geophys. Res., 88, 3072-3078, 1983.

Helliwell, R. A. and J. P. Katsufrakis, VLF wave injection into the magnetosphere from Siple station, Antarctica, J. Geophys. Res., 79, 2511-2518, 1974.

MATSUmото, H., Nonlinear whistler-model interaction and triggered emissions in the magnetosphere: a review, in Wave Instabilities in Space Plasma, edited by P. J. Palmadesso and K. Papadopoulos, pp. 163-190, D. Reidel Pub. Co., 1979.

Matsumoto, H., and Y. OMURA, Computer simulation studies of VLF triggered emissions: Deformation of distribution function by trapping and detrapping,. Geophys. Res. Lett., 10, 607-610, 1983.

Omura, Y. and H. MATSumoto, Computer simulations of basic processes of coherent whistler wave-particle interactions in the magnetosphere, J. Geophys. Res., 87, 4435-4444, 1982.

Roux, A. and R. Pellat, A theory of triggered emissions, J. Geophys. Res., 83, 1422-1441, 1978.

VOMVORIDIS, J. L., Nonuniform whistler mode propagation, Ph. D. thesis, Northwestern Univ., Evanston, I11., 1978. 